\title{
Atmospheric neutrino oscillations with Super-Kamiokande and prospects for SuperK-Gd
}

\section{Pablo Fernández Menéndez ${ }^{a, 1, *}$ on behalf of the Super-Kamiokande Collaboration} (a complete list of authors can be found at the end of the proceedings)

a University of Liverpool,

Department of Physics, Liverpool, United Kingdom

E-mail: pablofer@liverpool.ac.uk

The Super-Kamiokande (SK) experiment is a 50 kton water-Cherenkov detector located in Kamioka, Japan. Instrumented with more than 11,000 PMTs, it has been collecting data since 1996 and has been responsible for the very first observation of neutrino oscillations through the analysis of atmospheric neutrinos. Nowadays, the analysis of SK atmospheric neutrino data keeps providing some of the most precise measurements for neutrino oscillation parameters such as $\theta_{23}$, the neutrino mass ordering, $\Delta m_{32}^{2}$, and, to a lesser extent, the $\delta_{C P}$ phase.

In this presentation, an overview of the most recent atmospheric neutrino oscillation analysis results will be given, as well as, a glimpse of what is to come, concerning atmospheric neutrinos, in the recently started Gd-doped phase of the detector. This detector upgrade (SuperK-Gd), provides an efficient neutron tagging via Gd-neutron capture, potentially enhancing the sensitivity of the atmospheric neutrino oscillation analysis.

$37^{\text {th }}$ International Cosmic Ray Conference (ICRC 2021)

July 12th-23rd, 2021

Online - Berlin, Germany

\footnotetext{
*Presenter
} 


\section{Introduction}

In this contribution we show the analysis the whole atmospheric data from the first four phases of the Super-Kamiokande (Super-K) experiment (SK-I to SK-IV), which amounts for a total of 6044 days. The sensitivity of Super-K is not sufficient to precisely measure the remaining unknown mixing parameters. In particular we are limited by low statistics and difficult event classification in the multi-GeV sample, sensitive to the neutrino mass ordering.

The analysis as well as the upgrades introduced are optimized for sensitivity to the mass hierarchy. In this occasion, we present results without constraints from external experiments, except for the solar parameters which are fixed to the current best fit values.

In 2 and 3 atmospheric neutrinos and neutrino oscillations are briefly reviewed before describing the Super-K detector in 4 and the atmospheric neutrino analysis details and upgrades in 5. In 6 the results obtained from the analysis of the atmospheric neutrino data are presented and interpreted. The additional future upgrades being developed for Super-K's atmospheric neutrino analysis are presented in 7 and the prospect and status of SuperK-Gd in 8, finally concluding in 9.

\section{Atmospheric neutrino flux}

Atmospheric neutrinos are produced in the upper layers of the atmosphere and due to the interaction of cosmic rays with the present nitrogen and oxygen atoms. These interactions generate particles from which some of them eventually, decay into neutrinos. The main contribution to the production of atmospheric neutrinos, especially at sub-GeV energies are charged pions and kaons.

$$
\pi^{+} \rightarrow \mu^{+} v_{\mu} \rightarrow e^{+} v_{e} \overline{v_{\mu}} v_{\mu}
$$

Both, muon and electron (anti)neutrinos are produced at an approximate rate of two to one respectively below $1 \mathrm{GeV}$. That ratio between neutrino species increases at larger ener-

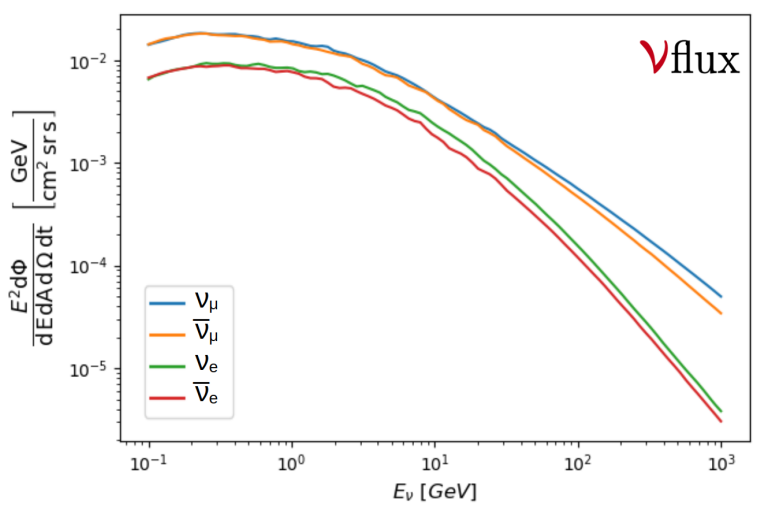

Figure 1: Unoscillated atmospheric $v_{\mu}$ (blue), $\overline{v_{\mu}}$ (orange), $v_{e}$ (green) and $\overline{v_{e}}$ (red) fluxes for the HKKM2014 model at Super-Kamiokande site as a function of the true neutrino energy [8], [1]. gies where kaon and charmed hadron decays dominate the atmospheric neutrino production. Atmospheric neutrinos are produced constantly and in large quantities spread over a vast range of energies, from tens of $\mathrm{MeV}$ up to hundreds of $\mathrm{TeV}$. Neutrinos are capable of traversing the Earth without interacting, which enables a wide range of propagation baselines before reaching the detector, from tens of kilometers to tens of thousands of kilometers and largely correlated with their zenith angle.

In the study of atmospheric neutrinos, detailed and comprehensive models and simulations are used, accounting for the primary cosmic ray flux, the hadron interaction and production, height of production and geomagnetic and solar activity effects among others. 
Furthermore, neutrino oscillations are sensitive not only to the baseline, but also to the different Earth's density layers through the MSW effect [11]. This includes an additional term in the propagation Hamiltonian and provides the scenario for having a fraction of tau neutrinos in the atmospheric neutrino flux at the detector. Precisely, neutrino oscillations were discovered by Super-K in 1998 through the deficit of upward-going $v_{\mu}$ in the atmospheric neutrino flux prediction and due to their oscillation to undetectable $v_{\tau}$ (disappearance channel) [7]. The atmospheric neutrino flux has been measured by Super-K and several other experiments, showing good agreement, within experimental uncertainty, with the theoretical models assuming oscillations between $100 \mathrm{MeV}$ and $100 \mathrm{TeV}$ and for muon and electron neutrinos,

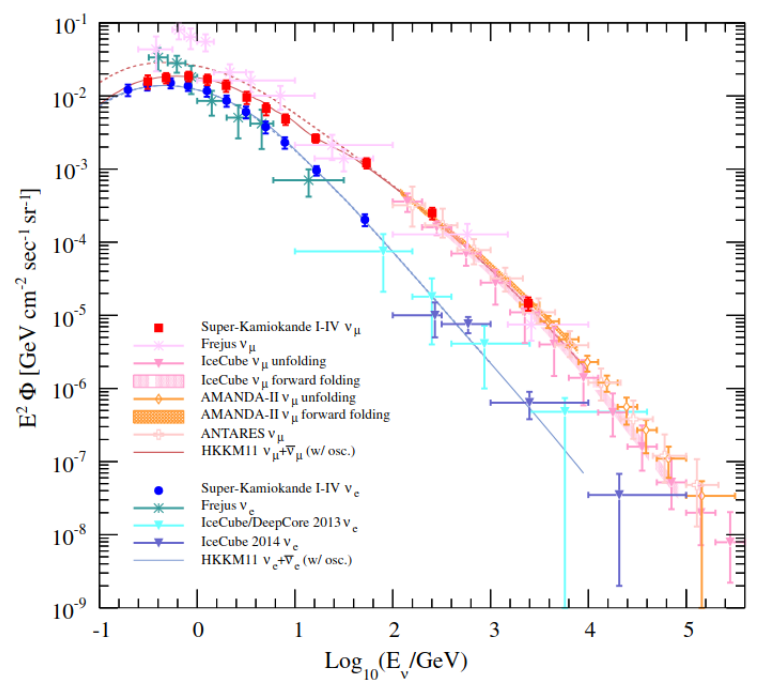

Figure 2: Measured energy spectra of the atmospheric $v_{e}$ and $v_{\mu}$ fluxes from various experiments [12], compared with the HKKM2011 model [9] with (solid) and without (dashed) oscillations. as shown in 2 .

\section{Neutrino oscillations}

Neutrinos oscillate because their eigenstates of the weak interaction (production and detection, $v_{l}$ ) are different from the neutrino mass eigenstates (propagation, $v_{i}$ ).

$$
\left|v_{l}\right\rangle=U_{P M N S}^{l i}\left|v_{i}\right\rangle,
$$

Where $U_{P M N S}$ corresponds to the Pontecorvo-Maki-Nakagawa-Sakata (PMNS) matrix, [10].

$$
U_{P M N S}=\left(\begin{array}{ccc}
c_{13} c_{12} & c_{13} s_{12} & s_{13} e^{-i \delta} \\
-c_{23} s_{12}-s_{23} s_{13} c_{12} e^{i \delta} & c_{23} c_{12}-s_{23} s_{13} s_{12} e^{i \delta} & s_{23} c_{13} \\
s_{23} s_{12}-c_{23} s_{13} c_{12} e^{i \delta} & -s_{23} c_{12}-c_{23} s_{13} s_{12} e^{i \delta} & c_{23} c_{13}
\end{array}\right)
$$

In addition to the four parameters from the PMNS matrix, the neutrino oscillation probability depends on other three parameters, the two possible squared mass differences $\left(\Delta m_{32}^{2}\right.$ and $\left.\Delta m_{21}^{2}\right)$ and the neutrino mass ordering ( $\operatorname{sign}$ of $\Delta m_{32}^{2}$ ), and is a function of the ratio of distance over neutrino en$\operatorname{ergy}\left(L / E_{v}\right)$. For a full description of the neutrino oscillations formalism, the reader may refer to [6].

Atmospheric neutrinos cover a wide range of $\frac{L}{E}$ and experience vacuum $\left(\cos \theta_{z e n}>0\right)$ and matter $\left(\cos \theta_{z e n}<0\right)$ oscillations. The latter change the effective propagation Hamiltonian of the neutrinos adding a new term from the coherent forward elastic scattering with the electrons present in the matter they propagate through.

$$
H=H_{v a c}+V_{M S W}=H_{v a c} \pm \sqrt{2} G_{F} N_{e} \operatorname{diag}(1,0,0)
$$


$H_{v a c}$ is the neutrino Hamiltonian for vacuum propagation and $V_{M S W}$ is the additional potential due to matter effects. This potential is proportional to the Fermi's constant, $G_{F}$, and to the Earth's electron density. The sign of the MSW term is positive for neutrinos and negative for antineutrinos and depends on the mass ordering.

The different features exhibited by atmospheric neutrino oscillations are summarized in 3 .
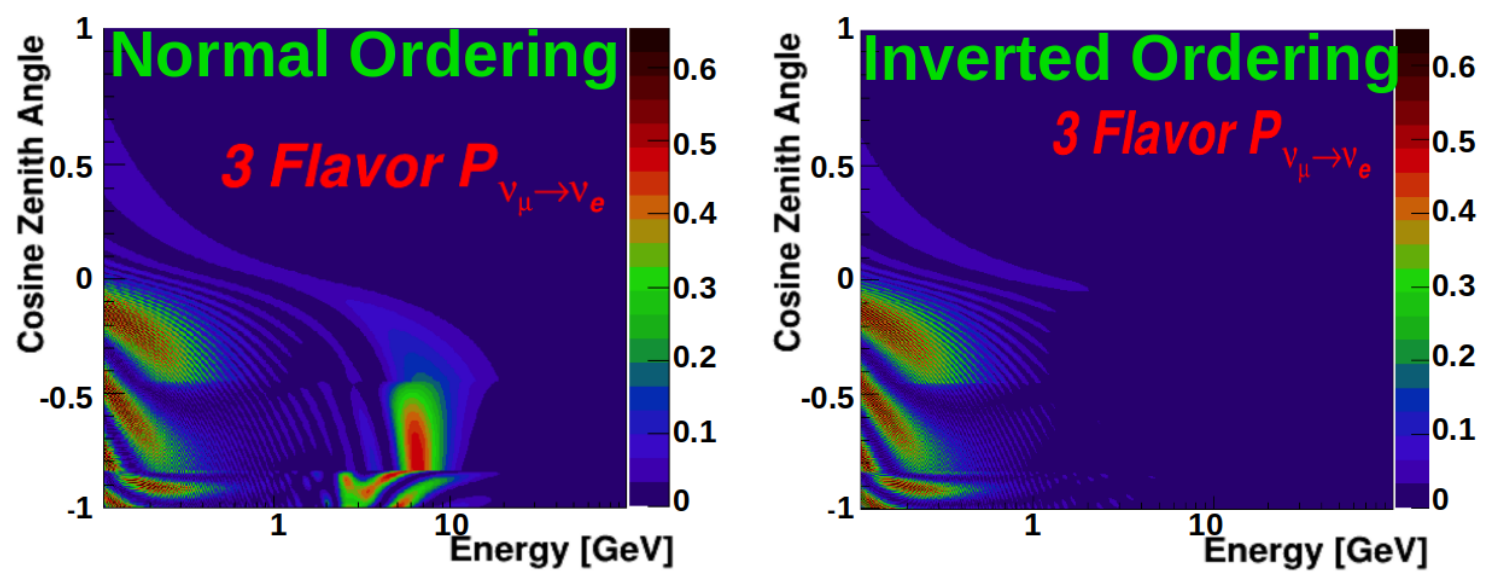

Figure 3: Two-dimensional oscillation probability for the $v_{\mu}$ to $v_{e}$ channel as function of zenith angle and neutrinos energy, and for both neutrino mass orderings, [13].

Oscillations of neutrinos only propagating through the atmosphere show very mild effects, whereas neutrinos traversing the Earth present much richer behaviour due to the larger and more varied baselines and the matter effects.

For atmospheric neutrinos, the dominant effect is due to muon neutrino to tau neutrino oscillation, modulated by the $\theta_{23}$ mixing angle, especially at energies below $1 \mathrm{GeV}$. The non-zero value of $\theta_{13}$ enables the matter-effect resonances, being sensitive to the different regions of the inner Earth, core and mantle. In addition, there exists a modest sensitivity to the $\delta_{C P}$ phase around the $1 \mathrm{GeV}$ region, although hard to disentangle from the effects of other parameters.

To overcome the present lack of statistics, in Super-K we are focused on improving the classification to discern between neutrinos and antineutrinos to improve the sensitivity to the ordering and the CP-phase.

\section{The Super-Kamiokande experiment}

The SK experiment consists in a water-Cherenkov detector located under Mt. Ikenoyama in Kamioka, Japan. It consists in a cylindrical tank containing $50 \mathrm{kton}$ of ultra-pure water, which currently and since 2020 is doped with gadolinium sulfate. It is optically divided into inner (ID) and outer (OD) detectors. The former instrumented with more than 11,000 inward-facing 20"-PMTs reaching $40 \%$ photo-coverage and containing a target volume of $32 \mathrm{kton}$. The OD is a two meterthick cylindrical shell surrounding the ID and instrumented with 1,885 outward-facing 8"-PMTs. 
Since the start in 1996, Super-Kamiokande has gone through six data taking periods, SKI, -II, -III, -IV, -V and -VI. The main changes and key aspect of each phase are summarized in 1.

In this report, the whole atmospheric neutrino data from SK-I to SK-IV is analyzed. In 2019, the Super-K tank was drained, refurbished and prepared for the Gd-loaded phase of the experiment, SK-VI, which began on 2020 with a $0.011 \%$ gadolinium concentration and is still ongoing. This major upgrade will enable a very

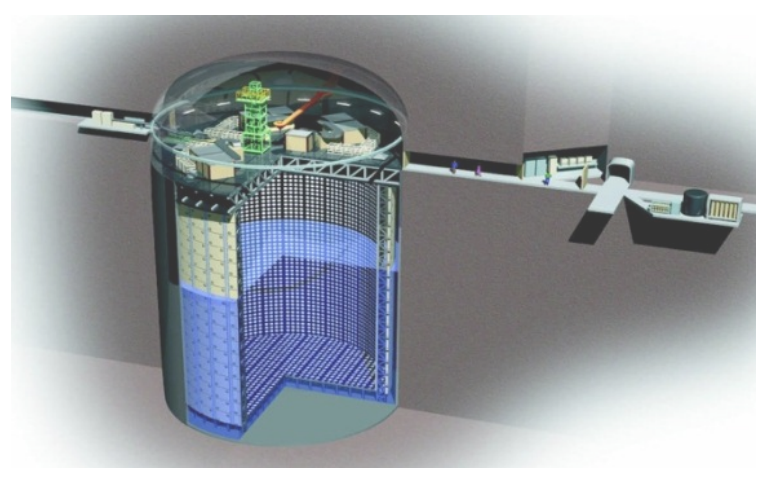

Figure 4: Drawing of the Super-Kamiokande experimental area. efficient neutron-tagging capabilities, improving the sensitivity to Diffuse Supernova Neutrino Background (DSNB), the background rejection for proton-decay searches and the distinction of neutrino and antineutrino at low and higher energies.

\begin{tabular}{||c|c|c||}
\hline SK phase & Period & Event \\
\hline \hline SK-I & $1996-2001$ & Start \\
\hline SK-II & $2003-2005$ & $20 \%$ PMT coverage after accident \\
\hline SK-III & $2006-2008$ & Resume 40\% PMT coverage \\
\hline SK-IV & $2008-2018$ & Electronics upgrade \\
\hline SK-V & $2019-2020$ & Upgrade for Gd-loading \\
\hline SK-VI & $2020-$ & $0.011 \%$ Gd-doped WC detector \\
\hline
\end{tabular}

Table 1: Dates and features of the six different data-taking periods of Super-Kamiokande.

\section{SK Atmospheric neutrinos}

Neutrino interactions which produce charged particles above the Cherenkov threshold in water are reconstructed based on the observed ring patterns projected on the detector walls. The PMT timing information is used to reconstruct the initial interaction vertex after correcting for the photon time of flight and the momentum of each ring is reconstructed based on the charge collected. The direction of each reconstructed particle is estimated based on the shape of its ring pattern. Energy and direction of the neutrino used in the oscillation analysis are then computed based on the reconstructed daughter particles.

\subsection{Event clasification}

In Super-K, atmospheric neutrino data are separated into three main categories, fully contained (FC), partially contained (PC) and upward-going muons (Up- $\mu$ ). Fully contained events have a reconstructed vertex within the 22.5 kton fiducial volume and no activity in the OD. Partially 
contained events have a reconstructed vertex within the fiducial volume but with some activity in the OD. Upward-going muons correspond to those neutrinos coming from below and interacting with the surrounding rock and producing a muon that leaves a signal in the detector.

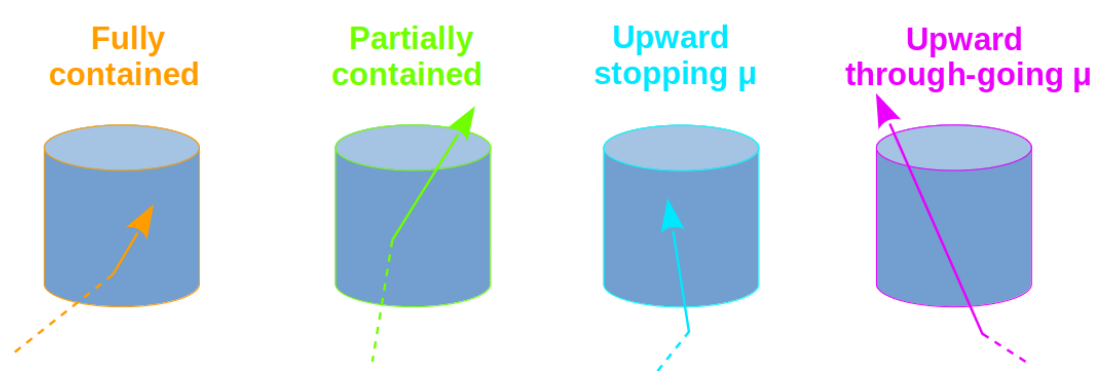

Figure 5: Schemes for the four different event topologies of Super-K's atmospheric neutrinos.

Attending to the ring pattern and opening angle, particles can be further classified into e-like (electrons and photons) with fuzzy edges and $\mu$-like (muons and charged pions) with crisp edges, as seen in the examples of 6
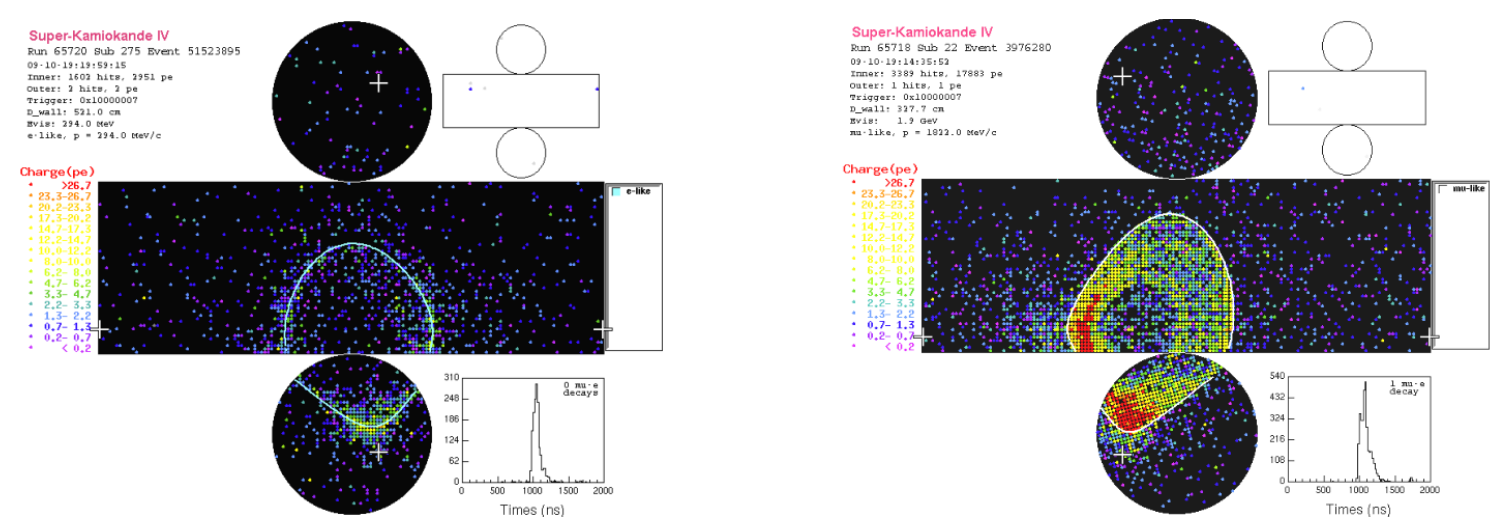

Figure 6: $e$-like (left) and $\mu$-like (right) Cherenkov ring patterns from Super-Kamiokande simulation.
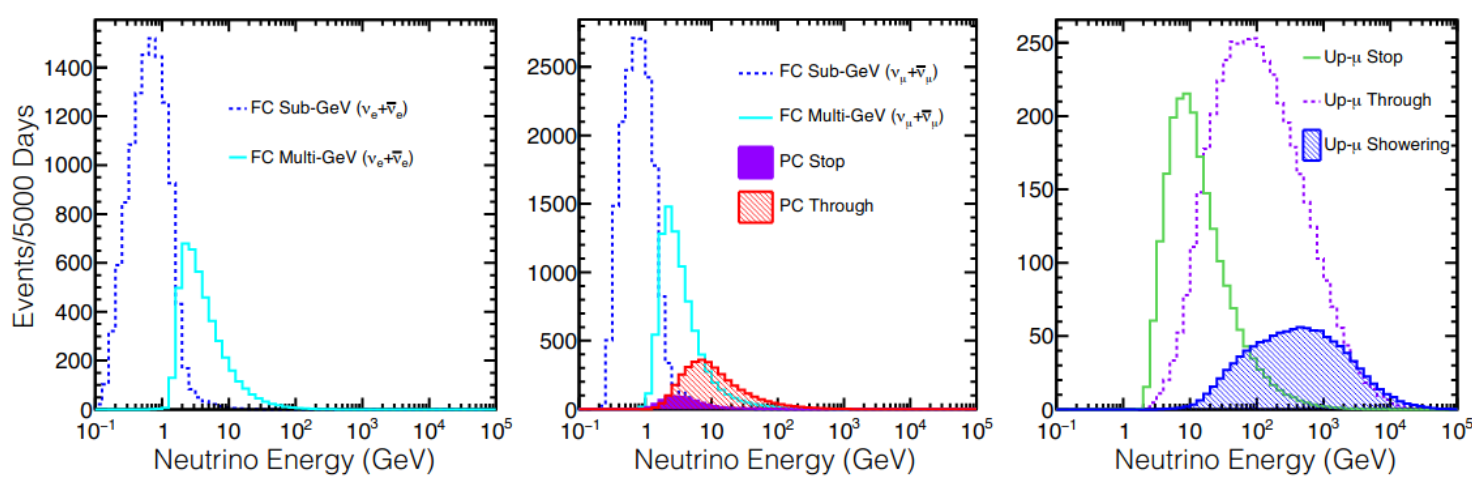

Figure 7: True Super-K atmospheric neutrino energy spectra from simulation without oscillations and split into the main event categories.

Fully contained events are the most populous and the ones which carry more information. This way, they are further divided into 14 subsamples. These are defined based on the number of 
rings, the reconstructed ID of the most energetic ring, total energy above or below $1 \mathrm{GeV}$, and other variables like the number of decay electrons or kinematic variables to distinguish $\mathrm{NC} \pi^{0}$ events and neutrinos from antineutrinos. Further details on the atmospheric neutrino classification can be found at [3].

\subsection{Oscillation analysis upgrades}

The present analysis includes relevant and significant upgrades as compared to the published results in [3].

The upgrade with the largest impact in the analysis is the usage of neutron tagging via hydrogen tagging, represented in 8 . Since SK-IV and thanks to the electronics upgrade, the $2.2 \mathrm{MeV}$ signal from the de-excitation of deuterium after hydrogen neutron-capture is detectable. Despite the low energy of the signal, a tagging efficiency of $25 \%$ is achieved. Neutrinos produce on average fewer neutrons than antineutrinos; then in addition to the usual cut in the number of electrons from muon decays for single-ring samples, a cut is applied in the number of tagged neutrons ( 0 neutrons or $>0$ neutrons) to improve the neutrino-antineutrino separation. This es-

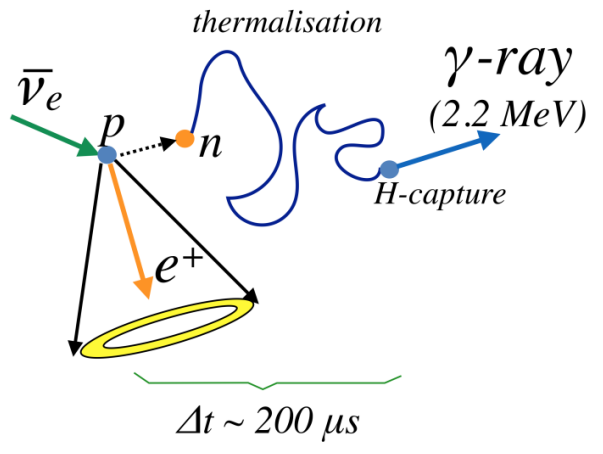

Figure 8: Scheme of the inverse- $\beta$ decay producing a e-like Cherenkov ring and a $2.2 \mathrm{MeV}$ photon from hydrogen neutron capture.

tablishes new sample definitions for the analysis of SK-IV data (hybrid analysis).

Another significant upgrade is the use of machine learning techniques (a boosted decission tree, BDT, in this case) to improve the classification of multi-ring events into the four usual samples: $\mathrm{CC} v_{e}, \mathrm{CC} \overline{v_{e}}, \mathrm{CC}\left(v_{\mu}+\overline{v_{\mu}}\right)$, and Other ( $\mathrm{NC}$ and $\left.\mathrm{CC} v_{\tau}\right)$. The implementation of this method increases the efficiency of the $\mathrm{CC} v_{e}$-like sample, from $34.4 \%$ to $46.7 \%$ while maintaining a similar purity.

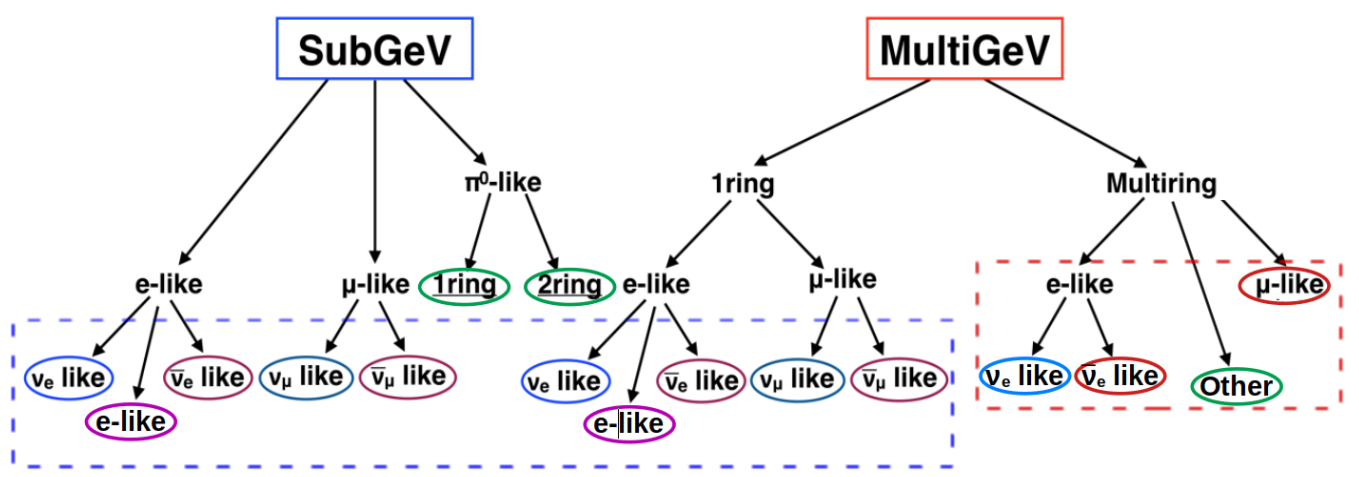

Figure 9: New sample definition for FC events in SK-IV. Dashed blue rectangle includes single-ring samples using cuts on the number of neutrons and electrons from muon decays. Dashed orange rectangle includes multi-ring samples using a BDT for the classification. 
Both of these updates have a significant impact in the purity and efficiency of the neutrino and antineutrino analysis samples and thus, in the sensitivity to the neutrino mass ordering and the CP-phase as shown in 10.

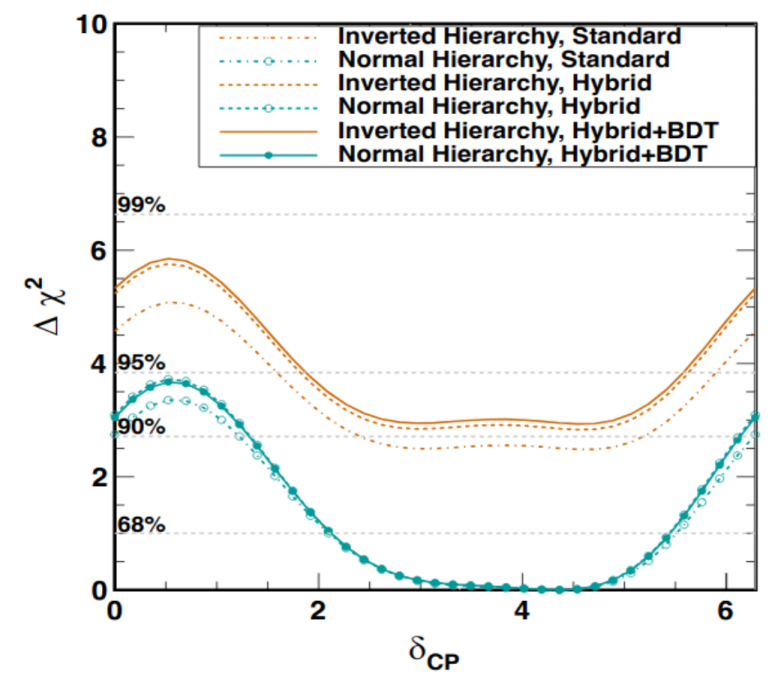

Figure 10: Comparison of the sensitivity studies for the $\delta_{C P}$ and for normal (cyan) and inverted (orange) neutrino mass orderings, assuming the Super-K exposure and for the standard (dash-dotted), hybrid (dashed) and hybrid with multi-ring BDT classification (solid) analyses.

Further updates in this analysis are the use of a new Monte Carlo simulation based on NEUT5.4 with updated interaction models, improved treatment and additional systematic and new zenith angle binning to better target separately the oscillation matter effects of core and mantle.

\section{Results from SK atmospheric neutrino oscillations analysis}

The data analyzed corresponds to 372 kton-year exposure, the full data set from the first four phases of SK. Results assume standard three flavor oscillations and only the solar parameters $\left(\theta_{12}\right.$ and $\Delta m_{21}^{2}$ ) are fixed.

Histograms in 11 and 12 show the good agreement existing between data and the Monte Carlo simulation prediction assuming the best fit mixing parameters. 

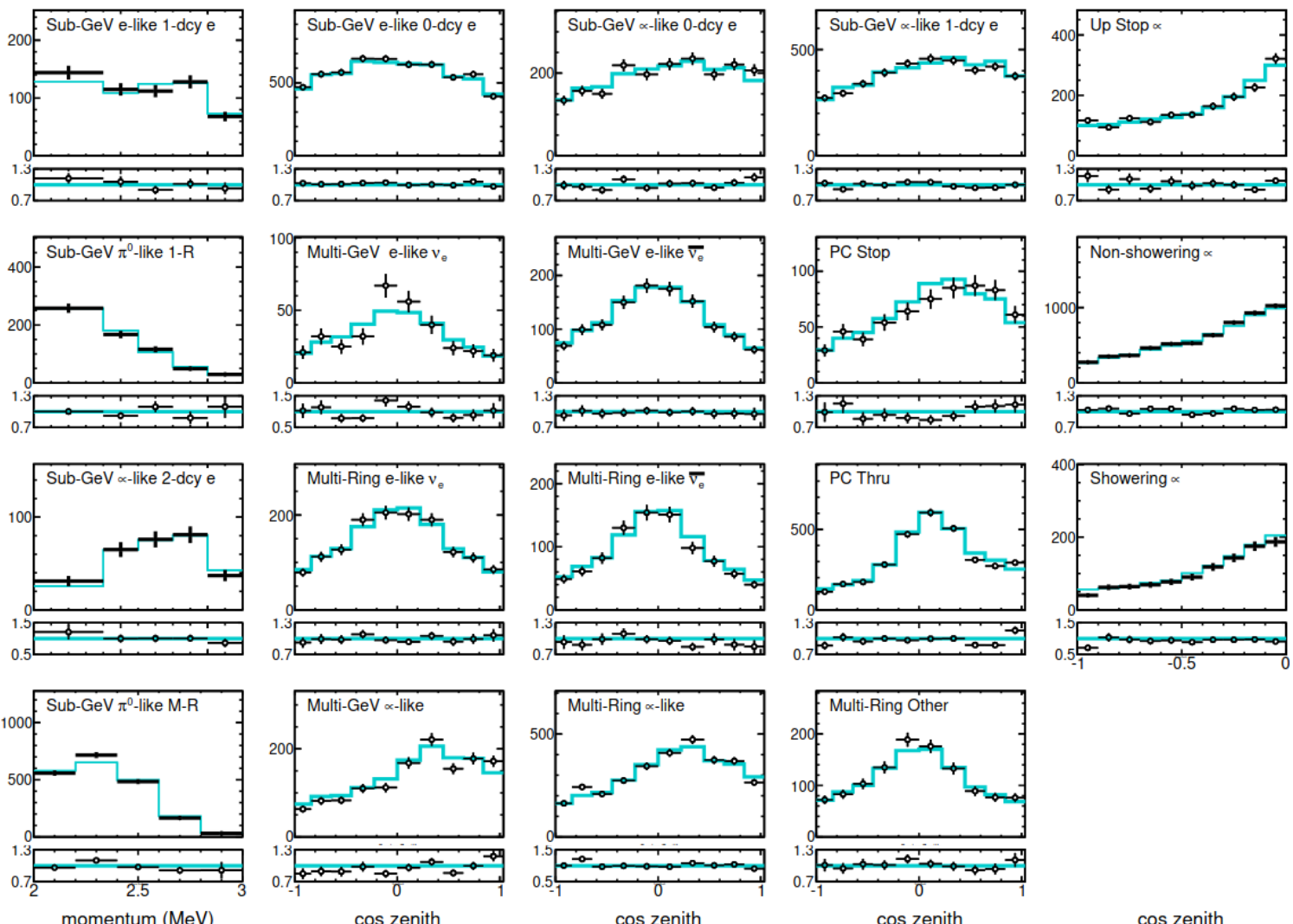

cos zenith

cos zenith

Figure 11: Data (black dots) with statistical error bars overlaid with the best fit simulation (cyan line) for the SK-I to SK-III samples and for the common samples across the four Super-K phases.
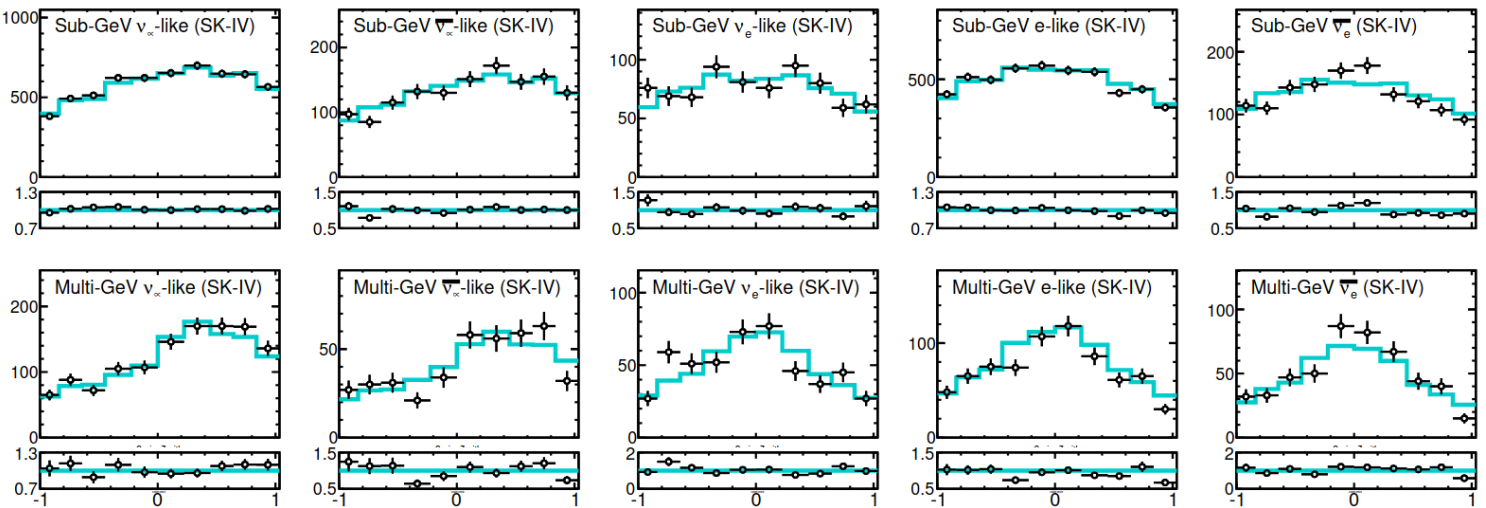

cos zenith

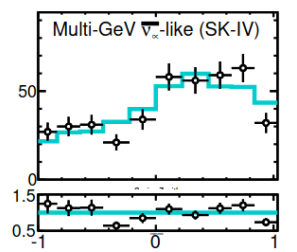

cos zenith

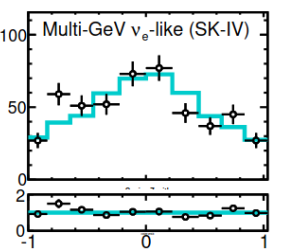

cos zenith

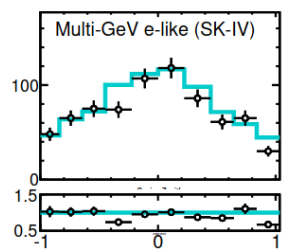

cos zenith

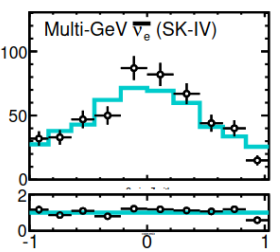

cos zenith

Figure 12: Data (black dots) with statistical error bars overlaid with the best fit simulation (cyan line) for the SK-IV additional samples.

Data are fit to the MC using a binned $\chi^{2}$ method built assuming Poisson statistics and incorporating systematic errors as scaling factors on the MC in each bin:

$$
\chi^{2}=2 \sum_{n}\left(E_{n}-O_{n}+O_{n} \ln \frac{O_{n}}{E_{n}}\right)+\sum_{i}\left(\frac{\epsilon_{i}}{\sigma_{i}}\right)^{2}
$$


Where $E_{n}$ and $O_{n}$ are the MC expected number of entries and data at the $n^{t h}$ bin, respectively. The second sum in 5 is the systematic errors penalization term, where $\sigma_{i}$ is the $1 \sigma$ variation of the $i^{t h}$ systematic error source and $\epsilon_{i}$ is the corresponding fitting parameter which satisfies $\partial \chi^{2} / \partial \epsilon_{i}=0$. This minimization is done for a grid of oscillation parameters, of which the one returning the lowest $\chi^{2}$ value is taken as the best fit.

Figure 13 shows the one-dimensional $\chi^{2}$ curves for all the free parameters of the fit. The analysis is done for both neutrino mass orderings independently. Results favor the first octant of $\theta_{23}$ and normal mass ordering and show a modest sensitivity to $\delta_{C P}$ with best fit value similar to that of T2K, [5]. Results also show some constraining power over $\theta_{13}$, with a non-zero best fit value consistent with reactor and long baseline experiments.
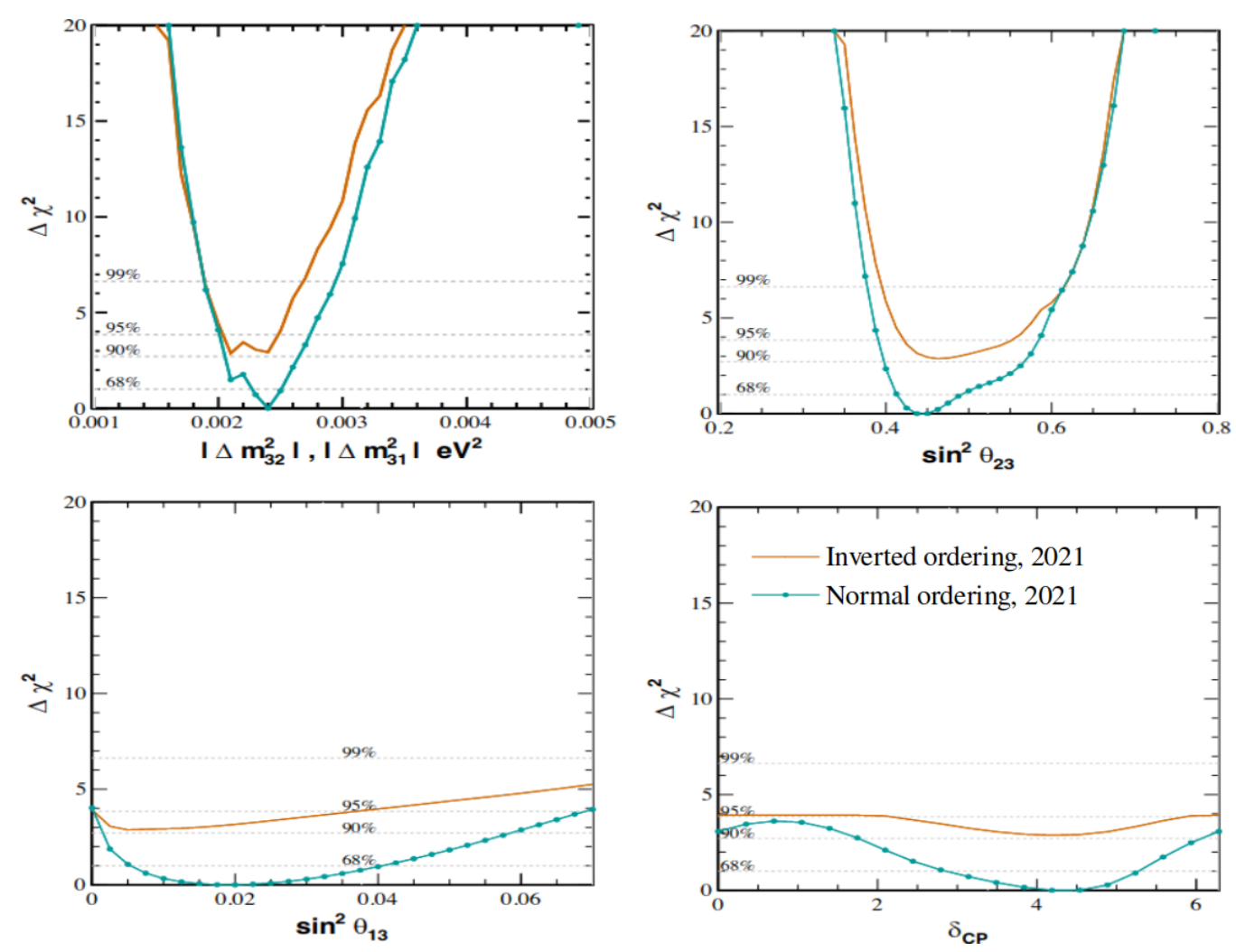

Figure 13: Constraints on neutrino oscillation parameters from the whole Super-K atmospheric neutrino data from SK-I to SK-IV. The offset between inverted ordering (orange lines) and normal ordering (cyan lines) corresponds to the difference in their minimum $\chi^{2}$ values.

Comparing these results with those previously (2018 analysis) released by Super-K, the octant preference has switched and the $1 \sigma$ interval is more constraining. This preference for the first octant is mild, being the former best fit value less than $2 \sigma$ away.

Normal mass ordering is still preferred although at a lower significance than in the previous analysis, $\chi_{I H}^{2}-\chi_{N H}^{2}=2.8$.

Finally, the sensitivity to both $\delta_{C P}$ and thet $a_{13}$ have improved due to the upgraded analysis and the increased statistics. 

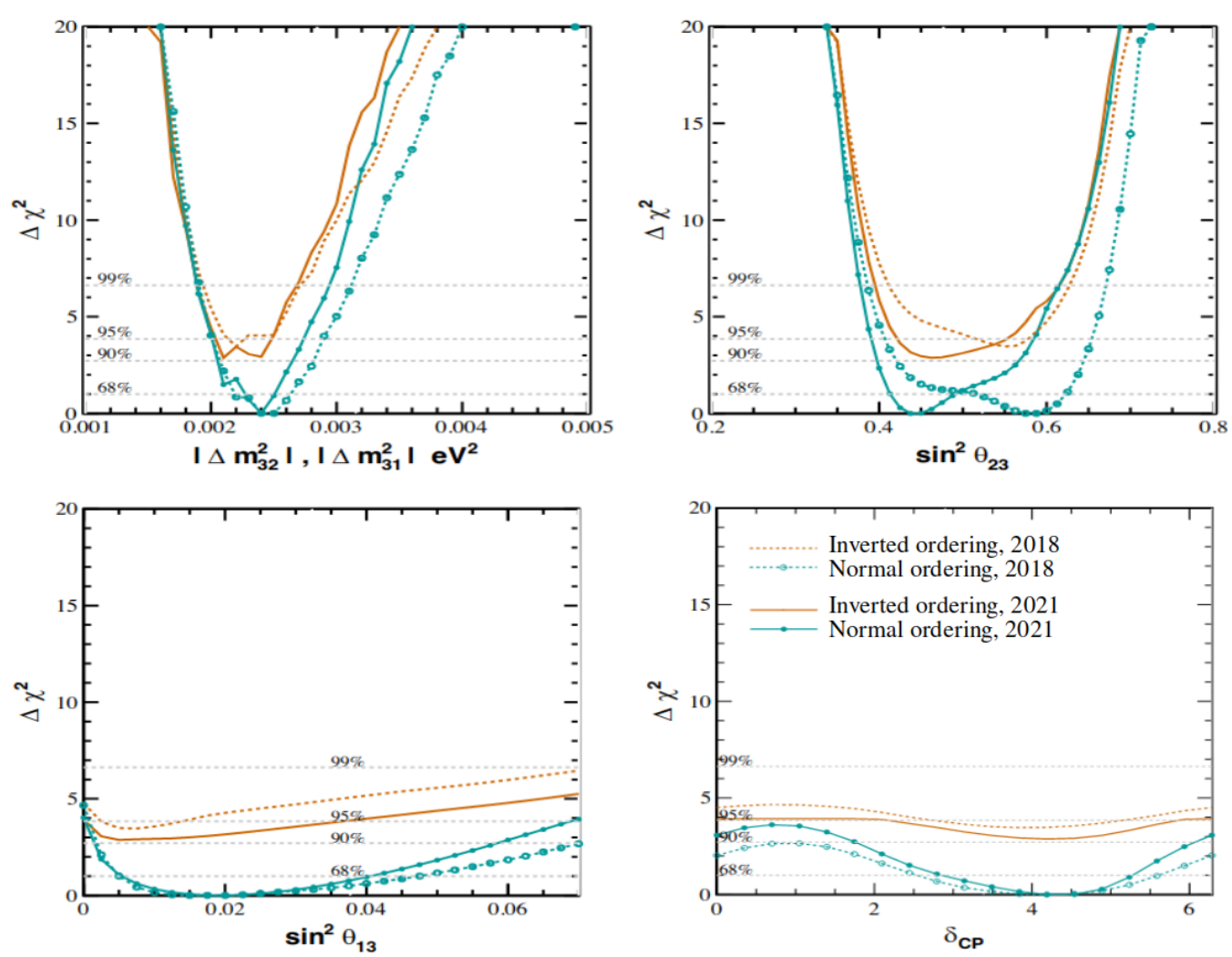

Figure 14: True Super-K atmospheric neutrino energy spectra from simulation without oscillations and split into the main event categories.

The $\chi^{2}$ maps for the Super-K 2021 results are publicly available at [2].

The preference for the normal mass hierarchy can be seen in more detail in 15 , where the $\chi^{2}$ difference for the best fit values assuming both orderings, show the most relevant samples for the fit. The significance resides in the purity of neutrino-like samples, improved by the described BDT-based multi-ring selection and neutron-based sample definition in SK-IV.

Similarly for $\delta_{C P}, 16$ shows the difference between the $\chi^{2}$ minima of two extreme values. In this case, the SK-IV Sub-GeV samples are those providing the most constraining power due to the impact of neutron tagging in the classification of neutrinos and antineutrinos. 


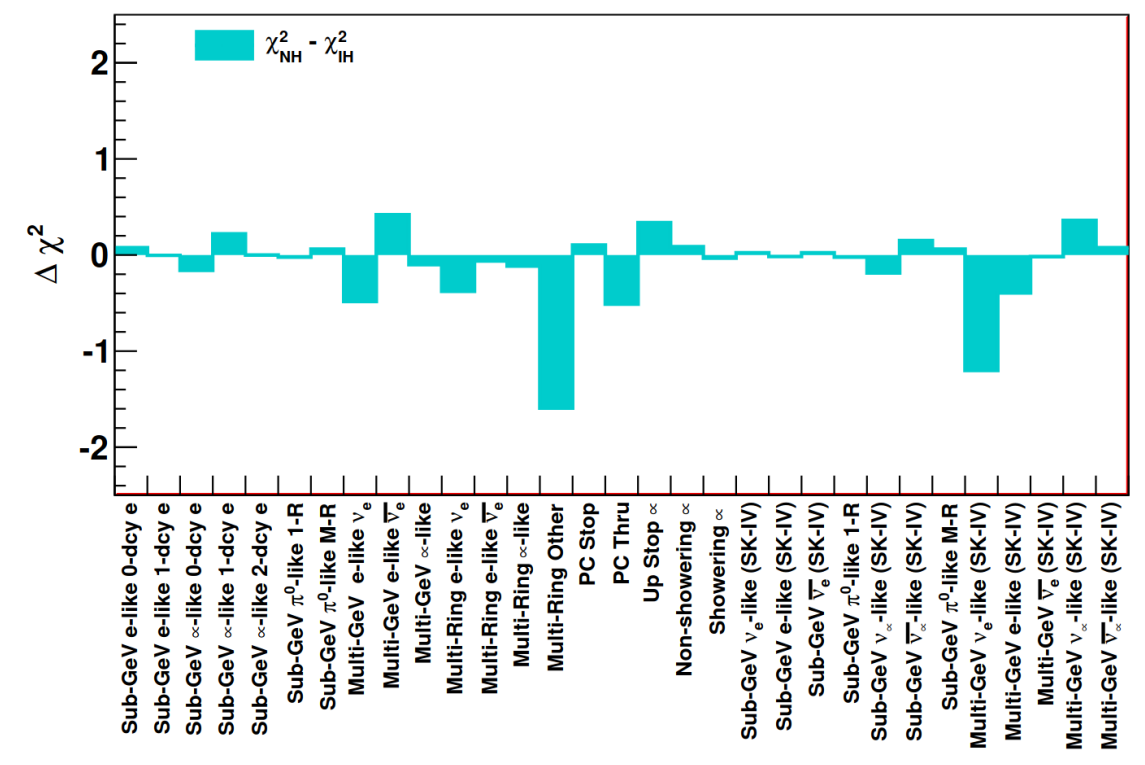

Figure 15: Sample by sample significance difference between both neutrino mass orderings (preference from data is normal).

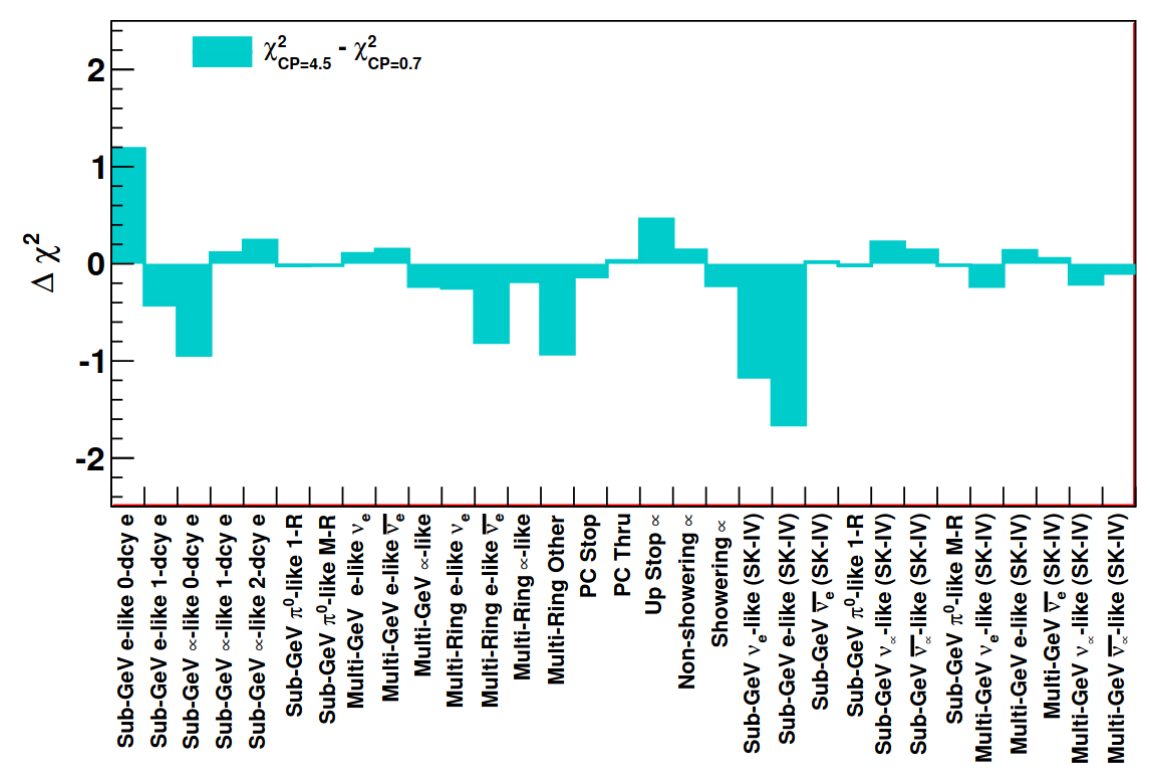

Figure 16: Sample by sample significance difference for two values of $\delta_{C P}, 4.5$ (close to the data best fit value, 4.1 ) and 0.7 .

\section{Future analysis upgrades}

Along with the presented set of updates in the atmospheric neutrino oscillation analysis, the Super-Kamiokande collaboration is working on various additional updates:

- Expand the fiducial volume from $2 \mathrm{~m}$ to $1 \mathrm{~m}$ away from the ID wall for all Super-K data taking periods. This will increase statistics by $20 \%$. Currently, studies are ongoing to validate the 
performance of the detector in this region.
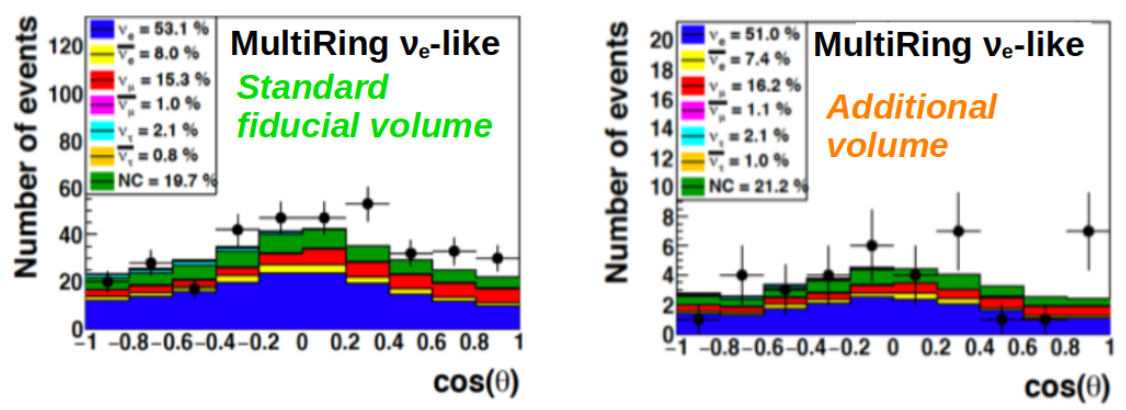

Figure 17: Zenith angle distribution of data (black dots) and MC (colored histograms) for the standard fiducial volume (reconstructed vertex $>2 \mathrm{~m}$ from the ID wall) on the left, and the planned fiducial volume expansion (reconstructed vertex between $1 \mathrm{~m}$ and $2 \mathrm{~m}$ from the ID wall ) on the right.

- Analysis of data from SK-V period.

- Include $v_{\tau}$ classification based on machine learning into the oscillation analysis.

- Improve the T2K model to the latest published result.

- Implementation of polynomial-based average density calculation for more accurate modelling of oscillations with matter effects.

\section{Prospects for SuperK-Gd atmospheric neutrinos}

The Gd-phase of Super-K began in July 2020, SK-VI, and since mid August the detector is running stably at $0.011 \%$ gadolinium concentration. This means that compared with the SK-IV, the neutron tagging efficiency has doubled (25\% to 50\%) and the neutron capture time reduced from $200 \mu$ s to $116 \mu$ s. All details regarding the development and status of the first Gd-loading in Super-K can be found at [4].

The atmospheric neutrino data of SK-VI is being carefully checked, showing good agreement with the performance from previous phases as shown in 18 .

In the presented results, we have already seen some of the potential of neutron tagging. With larger efficiency, the differentiation between neutrino and antineutrino will improve. Besides, it will also allow to explore other advantages of neutron tagging, such as improving the energy reconstruction as neutrons provide information about the Cherenkov-invisible energy of the neutrino interaction, and providing additional power to discriminate charged-current from neutral-current neutrino events. 

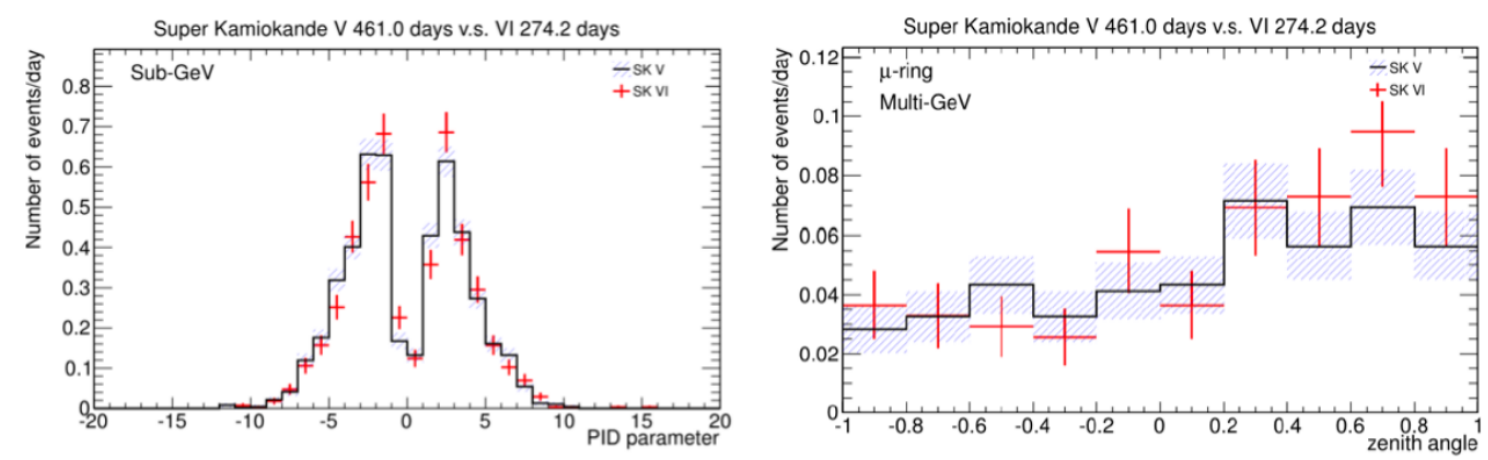

Figure 18: First reconstructed atmospheric neutrino data from the $6^{\text {th }}$ phase of Super-K (SK-VI).

\section{Conclusion}

Updated analysis of Super-Kamiokande atmospheric neutrino data over a 372 kton-year exposure of the detector indicates a weak preference for the normal mass ordering $\left(\Delta \chi^{2}=2.8\right)$. Results also indicate a slight preference for the first octant of $\theta_{23}$, a large value of $\delta_{C P}$ and a non-zero value of $\theta_{13}$.

Additionally, the upgrades included in the current oscillation analysis and the future plans regarding the Super-K and Superk-Gd atmospheric neutrino oscillation analysis are reviewed.

\section{References}

[1] Nuflux code, https://github.com/icecube/nuflux.

[2] $\chi^{2}$-maps for the super-kamiokande atmospheric neutrino oscillation results, https://indicosk.icrr.u-tokyo.ac.jp/event/5517/, 2021.

[3] K. Abe, C. Bronner, Y. Haga, Y. Hayato, M. Ikeda, K. Iyogi, J. Kameda, Y. Kato, Y. Kishimoto, L. Marti, and et al. Atmospheric neutrino oscillation analysis with external constraints in super-kamiokande i-iv. Physical Review D, 97(7), Apr 2018.

[4] K. Abe, C. Bronner, Y. Hayato, K. Hiraide, et al. First gadolinium loading to super-kamiokande. 2021.

[5] T. T. Collaboration. Constraint on the matter-antimatter symmetry-violating phase in neutrino oscillations. Nature, (580):339-344, 2020.

[6] G. Fantini, A. G. Rosso, F. Vissani, and V. Zema. The formalism of neutrino oscillations: an introduction, 2020.

[7] Y. Fukuda, T. Hayakawa, E. Ichihara, K. Inoue, K. Ishihara, H. Ishino, Y. Itow, T. Kajita, J. Kameda, S. Kasuga, and et al. Evidence for oscillation of atmospheric neutrinos. Phys. Rev. Lett., 81:1562-1567, Aug 1998.

[8] M. Honda, M. S. Athar, T. Kajita, K. Kasahara, and S. Midorikawa. Atmospheric neutrino flux calculation using the nrlmsise-00 atmospheric model. Physical Review D, 92(2), Jul 2015. 
[9] M. Honda, T. Kajita, K. Kasahara, and S. Midorikawa. Improvement of low energy atmospheric neutrino flux calculation using the jam nuclear interaction model. Physical Review D, 83(12), Jun 2011.

[10] Z. Maki, M. Nakagawa, and S. Sakata. Remarks on the Unified Model of Elementary Particles. Progress of Theoretical Physics, 28(5):870-880, 111962.

[11] S. Mikheyev and A. Y. Smirnov. Resonance enhancement of oscillations in matter and solar neutrino spectroscopy. Yadernaya Fizika, 42:1441-1448, Jan. 1985.

[12] E. Richard, K. Okumura, K. Abe, Y. Haga, Y. Hayato, M. Ikeda, K. Iyogi, J. Kameda, Y. Kishimoto, M. Miura, and et al. Measurements of the atmospheric neutrino flux by superkamiokande: Energy spectra, geomagnetic effects, and solar modulation. Physical Review D, 94(5), Sep 2016.

[13] R. Wendell. Prob3++ code, https://github.com/rogerwendell/prob3plusplus. 


\section{The Super-Kamiokande Collaboration}

K. Abe,${ }^{1,45}$ C. Bronner, ${ }^{1}$ Y. Hayato, ${ }^{1,45}$ K. Hiraide,${ }^{1,45}$ M. Ikeda, ${ }^{1}$ J. Kameda, ${ }^{1,45}$ Y. Kanemura, ${ }^{1}$ Y. Kataoka, ${ }^{1}$ S. Miki, ${ }^{1}$ M. Miura, ${ }^{1,45}$ S. Moriyama, ${ }^{1,45}$ Y. Nagao, ${ }^{1}$ M. Nakahata, ${ }^{1,45}$ S. Nakayama,,${ }^{1,45}$ K. Okamoto, ${ }^{1}$ G. Pronost,${ }^{1}$ H. Sekiya, ${ }^{1,45}$ M. Shiozawa, ${ }^{1,45}$ Y. Sonoda, ${ }^{1}$ Y. Suzuki, ${ }^{1}$ A. Takeda,${ }^{1}{ }^{45}$ Y. Takemoto, ${ }^{1}$ A. Takenaka, ${ }^{1}$ H. Tanaka, ${ }^{1}$ S. Watanabe,${ }^{1}$ T. Yano, ${ }^{1}$

S. Han, ${ }^{2}$ T. Kajita,${ }^{2,45}$ K. Okumura,${ }^{2,45}$ T. Tashiro, ${ }^{2}$ J. Xia, ${ }^{2}$ G. D. Megias, ${ }^{3}$ L. Labarga, ${ }^{4}$ Ll. Marti, ${ }^{4}$ B. Zaldivar, ${ }^{4}$

B. W. Pointon, ${ }^{6,49}$ E. Kearns, ${ }^{5,45}$ J. L. Raaf, ${ }^{5}$ L. Wan, ${ }^{5}$ T. Wester, ${ }^{5}$ J. Bian, ${ }^{7}$ N. J. Griskevich, ${ }^{7}$ W. R. Kropp ${ }^{7}$ S. Locke, ${ }^{7}$

S. Mine, ${ }^{7}$ M. B. Smy, ${ }^{7,45}$ H. W. Sobel, ${ }^{7,45}$ V. Takhistov, ${ }^{7,45}$ J. Hill, ${ }^{8}$ J. Y. Kim, ${ }^{9}$ I. T. Lim,,${ }^{9}$ R. G. Park, ${ }^{9}$ B. Bodur, ${ }^{10}$

K. Scholberg, ${ }^{10,45}$ C. W. Walter,${ }^{10,45}$ L. Bernard,${ }^{11}$ A. Coffani,,${ }^{11}$ O. Drapier,${ }^{11}$ S. El Hedri, ${ }^{11}$ A. Giampaolo, ${ }^{11}$ M. Gonin, ${ }^{11}$

Th. A. Mueller, ${ }^{11}$ P. Paganini, ${ }^{11}$ B. Quilain, ${ }^{11}$ T. Ishizuka, ${ }^{12}$ T. Nakamura, ${ }^{13}$ J. S. Jang, ${ }^{14}$ J. G. Learned, ${ }^{15}$ S. Cao, ${ }^{16}$

L. H. V. Anthony, ${ }^{17}$ D. Martin, ${ }^{17}$ M. Scott, ${ }^{17}$ A. A. Sztuc,,${ }^{17}$ Y. Uchida, ${ }^{17}$ V. Berardi, ${ }^{18}$ M. G. Catanesi, ${ }^{18}$ E. Radicioni, ${ }^{18}$ N. F. Calabria, ${ }^{19}$ L. N. Machado, ${ }^{19}$ G. De Rosa,${ }^{19}$ G. Collazuol, ${ }^{20}$ F. Iacob,${ }^{20}$ M. Lamoureux,${ }^{20}$ M. Mattiazzi, ${ }^{20}$ N. Ospina, ${ }^{20}$

L. Ludovici, ${ }^{21}$ Y. Maekawa, ${ }^{22}$ Y. Nishimura, ${ }^{22}$ M. Friend, ${ }^{23}$ T. Hasegawa, ${ }^{23}$ T. Ishida, ${ }^{23}$ T. Kobayashi, ${ }^{23}$ M. Jakkapu, ${ }^{23}$

T. Matsubara, ${ }^{23}$ T. Nakadaira, ${ }^{23}$ K. Nakamura, ${ }^{23}{ }^{45}$ Y. Oyama, ${ }^{23}$ K. Sakashita, ${ }^{23}$ T. Sekiguchi, ${ }^{23}$ T. Tsukamoto, ${ }^{23}$ T. Boschi, ${ }^{24}$ F. Di Lodovico, ${ }^{24}$ J. Gao,${ }^{24}$ J. Migenda,${ }^{24}$ M. Taani, ${ }^{24}$ S. Zsoldos,${ }^{24}$ Y. Nakano, ${ }^{25}$ H. Ozaki, ${ }^{25}$ A. T. Suzuki, ${ }^{25}$ Y. Takeuchi, ${ }^{25,45}$ S. Yamamoto, ${ }^{25}$ Y. Kotsar, ${ }^{25}$ A. Ali, ${ }^{26}$ J. Feng, ${ }^{26}$ T. Kikawa, ${ }^{26}$ M. Mori, ${ }^{26}$ T. Nakaya,${ }^{26,45}$ R. A. Wendell,${ }^{26,45}$ K. Yasutome, ${ }^{26}$ P. Fernandez, ${ }^{27}$ N. McCauley, ${ }^{27}$ P. Mehta, ${ }^{27}$ K. M. Tsui, ${ }^{27}$ Y. Fukuda, ${ }^{28}$ Y. Itow, ${ }^{29,} 30$ H. Menjo, ${ }^{29}$ K. Sato, ${ }^{29}$

J. Lagoda, ${ }^{31}$ S. M. Lakshmi, ${ }^{31}$ P. Mijakowski, ${ }^{31}$ J. Zalipska, ${ }^{31}$ J. Jiang, ${ }^{32}$ C. K. Jung, ${ }^{32}$ C. Vilela, ${ }^{32}$ M. J. Wilking, ${ }^{32}$

C. Yanagisawa, ${ }^{32}$ M. Harada, ${ }^{33}$ H. Ishino, ${ }^{33}$ S. Ito, ${ }^{33}$ H. Kitagawa, ${ }^{33}$ Y. Koshio, ${ }^{33,45}$ W. Ma, ${ }^{33}$ N. Piplani,${ }^{33}$ S. Sakai, ${ }^{33}$

G. Barr, ${ }^{34}$ D. Barrow, ${ }^{34}$ L. Cook,${ }^{34,45}$ A. Goldsack, $,{ }^{34}, 45$ S. Samani, ${ }^{34}$ D. Wark, ${ }^{34,}{ }^{39}$ F. Nova, ${ }^{35}$ J. Y. Yang, ${ }^{36}$ S. J. Jenkins, ${ }^{37}$

M. Malek, ${ }^{37}$ J. M. McElwee, ${ }^{37}$ O. Stone,${ }^{37}$ M. D. Thiesse, ${ }^{37}$ L. F. Thompson, ${ }^{37}$ H. Okazawa, ${ }^{38}$ S. B. Kim,${ }^{40}$ I. Yu ${ }^{40}$

A. K. Ichikawa, ${ }^{41}$ K. Nakamura, ${ }^{41}$ K. Nishijima, ${ }^{42}$ M. Koshiba,${ }^{43,}{ }^{*}$ K. Iwamoto, ${ }^{44}$ Y. Nakajima, ${ }^{44}$ N. Ogawa, ${ }^{44}$

M. Yokoyama ${ }^{44,45}$ K. Martens, ${ }^{45}$ M. R. Vagins,${ }^{45,7}$ M. Kuze,${ }^{46}$ S. Izumiyama ${ }^{46}$ M. Inomoto,${ }^{47}$ M. Ishitsuka ${ }^{47}$ H. Ito, ${ }^{47}$

T. Kinoshita, ${ }^{47}$ R. Matsumoto, ${ }^{47}$ M. Shinoki, ${ }^{47}$ T. Suganuma, ${ }^{47}$ J. F. Martin, ${ }^{48}$ H. A. Tanaka, ${ }^{48}$ T. Towstego, ${ }^{48}$ R. Akutsu, ${ }^{49}$ P. de Perio, ${ }^{49}$ M. Hartz ${ }^{49}$ A. Konaka, ${ }^{49}$ N. W. Prouse, ${ }^{49}$ S. Chen,${ }^{50}$ B. D. Xu, ${ }^{50}$ M. Posiadala-Zezula, ${ }^{51}$ D. Hadley, ${ }^{52}$ M. O'Flaherty, ${ }^{52}$ B. Richards,${ }^{52}$ B. Jamieson, ${ }^{53}$ J. Walker, ${ }^{53}$ A. Minamino, ${ }^{54}$ G. Pintaudi, ${ }^{54}$ S. Sano, ${ }^{54}$ and R. Sasaki ${ }^{54}$ (The Super-Kamiokande Collaboration)

${ }^{1}$ Kamioka Observatory, Institute for Cosmic Ray Research, University of Tokyo, Kamioka, Gifu 506-1205, Japan

${ }^{2}$ Research Center for Cosmic Neutrinos, Institute for Cosmic Ray Research, University of Tokyo, Kashiwa, Chiba 277-8582, Japan

${ }^{3}$ Institute for Cosmic Ray Research, University of Tokyo, Kashiwa, Chiba 277-8582, Japan

${ }^{4}$ Department of Theoretical Physics, University Autonoma Madrid, 28049 Madrid, Spain ${ }^{5}$ Department of Physics, Boston University, Boston, MA 02215, USA

${ }^{6}$ Department of Physics, British Columbia Institute of Technology, Burnaby, BC, V5G 3H2, Canada

${ }^{7}$ Department of Physics and Astronomy, University of California, Irvine, Irvine, CA 92697-4575, USA

${ }^{8}$ Department of Physics, California State University, Dominguez. Hills, Carson, CA 90747, USA

${ }^{9}$ Institute for Universe and Elementary Particles, Chonnam National University, Gwangju 61186, Korea

${ }^{10}$ Department of Physics, Duke University, Durham NC 27708, USA

${ }^{11}$ Ecole Polytechnique, IN2P3-CNRS, Laboratoire Leprince-Ringuet, F-91120 Palaiseau, France

${ }^{12}$ Junior College, Fukuoka Institute of Technology, Fukuoka, Fukuoka 811-0295, Japan ${ }^{13}$ Department of Physics, Gifu University, Gifu, Gifu 501-1193, Japan

${ }^{14}$ GIST College, Gwangju Institute of Science and Technology, Gwangju 500-712, Korea

${ }^{15}$ Department of Physics and Astronomy, University of Hawaii, Honolulu, HI 96822, USA

${ }^{16}$ Institute For Interdisciplinary Research in Science and Education, ICISE, Quy Nhon, 55121, Vietnam

${ }^{17}$ Department of Physics, Imperial College London, London, SW7 2AZ, United Kingdom

18 Dipartimento Interuniversitario di Fisica, INFN Sezione di Bari and Università e Politecnico di Bari, I-70125, Bari, Italy

${ }^{19}$ Dipartimento di Fisica, INFN Sezione di Napoli and Università di Napoli, I-80126, Napoli, Italy

${ }^{20}$ Dipartimento di Fisica, INFN Sezione di Padova and Università di Padova, I-35131, Padova, Italy

${ }^{21}$ INFN Sezione di Roma and Università di Roma "La Sapienza”, I-00185, Roma, Italy

${ }^{22}$ Department of Physics, Keio University, Yokohama, Kanagawa, 223-8522, Japan

${ }^{23}$ High Energy Accelerator Research Organization (KEK), Tsukuba, Ibaraki 305-0801, Japan

${ }^{24}$ Department of Physics, King's College London, London, WC2R 2LS, UK

${ }^{25}$ Department of Physics, Kobe University, Kobe, Hyogo 657-8501, Japan

${ }^{26}$ Department of Physics, Kyoto University, Kyoto, Kyoto 606-8502, Japan

${ }^{27}$ Department of Physics, University of Liverpool, Liverpool, L69 7ZE, United Kingdom

${ }^{28}$ Department of Physics, Miyagi University of Education, Sendai, Miyagi 980-0845, Japan

${ }^{29}$ Institute for Space-Earth Environmental Research, Nagoya University, Nagoya, Aichi 464-8602, Japan

${ }^{30}$ Kobayashi-Maskawa Institute for the Origin of Particles and the Universe, Nagoya University, Nagoya, Aichi 464-8602, Japan ${ }^{31}$ National Centre For Nuclear Research, 02-093 Warsaw, Poland 
${ }^{32}$ Department of Physics and Astronomy, State University of New York at Stony Brook, NY 11794-3800, USA

${ }^{33}$ Department of Physics, Okayama University, Okayama, Okayama 700-8530, Japan

${ }^{34}$ Department of Physics, Oxford University, Oxford, OX1 3PU, United Kingdom

${ }^{35}$ Rutherford Appleton Laboratory, Harwell, Oxford, OX11 OQX, UK

${ }^{36}$ Department of Physics, Seoul National University, Seoul 151-742, Korea

${ }^{37}$ Department of Physics and Astronomy, University of Sheffield, S3 7RH, Sheffield, United Kingdom

${ }^{38}$ Department of Informatics in Social Welfare, Shizuoka University of Welfare, Yaizu, Shizuoka, 425-8611, Japan

${ }^{39}$ STFC, Rutherford Appleton Laboratory, Harwell Oxford, and Daresbury Laboratory, Warrington, OX11 OQX, United Kingdom

${ }^{40}$ Department of Physics, Sungkyunkwan University, Suwon 440-746, Korea

${ }^{41}$ Department of Physics, Faculty of Science, Tohoku University, Sendai, Miyagi, 980-8578, Japan

${ }^{42}$ Department of Physics, Tokai University, Hiratsuka, Kanagawa 259-1292, Japan

${ }^{43}$ The University of Tokyo, Bunkyo, Tokyo 113-0033, Japan

${ }^{44}$ Department of Physics, University of Tokyo, Bunkyo, Tokyo 113-0033, Japan

${ }^{45}$ Kavli Institute for the Physics and Mathematics of the Universe (WPI), The University of

Tokyo Institutes for Advanced Study, University of Tokyo, Kashiwa, Chiba 277-8583, Japan

${ }^{46}$ Department of Physics, Tokyo Institute of Technology, Meguro, Tokyo 152-8551, Japan

${ }^{47}$ Department of Physics, Faculty of Science and Technology, Tokyo University of Science, Noda, Chiba 278-8510, Japan

${ }^{48}$ Department of Physics, University of Toronto, ON, M5S 1A7, Canada

${ }^{49}$ TRIUMF, 4004 Wesbrook Mall, Vancouver, BC, V6T2A3, Canada

${ }^{50}$ Department of Engineering Physics, Tsinghua University, Beijing, 100084, China

${ }^{51}$ Faculty of Physics, University of Warsaw, Warsaw, 02-093, Poland

${ }^{52}$ Department of Physics, University of Warwick, Coventry, CV4 7AL, UK

${ }^{53}$ Department of Physics, University of Winnipeg, MB R3J 3L8, Canada

${ }^{54}$ Department of Physics, Yokohama National University, Yokohama, Kanagawa, 240-8501, Japan

PACS numbers:

*Deceased. 